\title{
Long-Term Trend of Temperature Derived by Statistical Downscaling Based on EOF Analysis*
}

\author{
FAN Lijun ${ }^{1,2,3 \dagger}$ (范丽军), FU Congbin ${ }^{1,2}$ (符淙斌), and CHEN Deliang ${ }^{3}$ (陈德亮) \\ 1 Key Laboratory of Regional Climate-Environment Research for Temperate East Asia (RCE-TEA), \\ Institute of Atmospheric Physics, Chinese Academy of Sciences, Beijing 100029, China \\ 2 College of Atmospheric Sciences, Lanzhou University, Lanzhou 730000, China \\ 3 Department of Earth Sciences, University of Gothenburg, Göteborg 40530, Sweden
}

(Received October 14, 2009; in final form March 29, 2011)

\begin{abstract}
This study analyzes the ability of statistical downscaling models in simulating the long-term trend of temperature and associated causes at 48 stations in northern China in January and July 1961-2006. The statistical downscaling models are established through multiple stepwise regressions of predictor principal components (PCs). The predictors in this study include temperature at $850 \mathrm{hPa}(\mathrm{T} 850)$, and the combination of geopotential height and temperature at $850 \mathrm{hPa}(\mathrm{H} 850+\mathrm{T} 850)$. For the combined predictors, Empirical Orthogonal Function (EOF) analysis of the two combined fields is conducted. The modeling results from HadCM3 and ECHAM5 under 20C3M and SERS A1B scenarios are applied to the statistical downscaling models to construct local present and future climate change scenarios for each station, during which the projected EOF analysis and the common EOF analysis are utilized to derive EOFs and PCs from the two general circulation models (GCMs). The results show that (1) the trend of temperature in July is associated with the first EOF pattern of the two combined fields, not with the EOF pattern of the regional warming; (2) although HadCM3 and ECHAM5 have simulated a false long-term trend of temperature, the statistical downscaling method is able to well reproduce a correct long-term trend of temperature in northern China due to the successful simulation of the trend of main PCs of the GCM predictors; (3) when the two-field combination and the projected EOF analysis are used, temperature change scenarios have a similar seasonal variation to the observed one; and (4) compared with the results of the common EOF analysis, those of the projected EOF analysis have been much more strongly determined by the observed large-scale atmospheric circulation patterns.
\end{abstract}

Key words: long-term trend of temperature, statistical downscaling, projected EOF analysis, common EOF analysis, northern China

Citation: Fan Lijun, Fu Congbin, and Chen Deliang, 2011: Long-term trend of temperature derived by statistical downscaling based on EOF analysis. Acta Meteor. Sinica, 25(3), 327-339, doi: 10.1007/s13351-011-0308-0.

\section{Introduction}

Global climate change may lead to changes in human environment; especially, regional climate change may have an important effect on the local human and natural system. Therefore, it is important to assess and project the regional climate change. Although the major concern nowadays is global warming, a surface cooling trend from spring to summer has been observed in the central eastern China since the 1950s. $\mathrm{Hu}$ et al. (2003) found that pronounced warming is observed in China in winter, spring, and autumn, particularly in the northern part of China; however, a cooling trend in central China occurs interestingly in summer. It is well known that GCMs are primary tools to reproduce the present global climate change and project future changes owing to the continuing increase of greenhouse gas concentration in the atmosphere. GCMs are not generally designed for local or regional climate change studies due to coarse resolution. Therefore, in many climate change impact studies, there is a need to convert the GCM outputs

* Supported by the National Natural Science Foundation of China (40705030), Knowledge Innovation Project (KZCX2-EW-202) and Strategic Priority Research Program (XDA05090103) of the Chinese Academy of Sciences.

${ }^{\dagger}$ Corresponding author: fanlj@tea.ac.cn.

(C)The Chinese Meteorological Society and Springer-Verlag Berlin Heidelberg 2011 
into high-resolution climate scenarios on the regional scale. Two main approaches: dynamical and statistical downscaling, are used for this purpose.

Dynamical downscaling uses high-resolution climate models to predict or hindcast climate changes over the globe or regional sub-domains. Either observed or lower-resolution GCM data are used as boundary conditions. Dynamical downscaling has the potential to capture mesoscale nonlinear effects and to provide coherent information among multiple climate variables. These models are formulated using physical principles and they can credibly reproduce a broad range of climates around the world. The main drawbacks of dynamical models are their computational cost and that in future climates the parameterization schemes they use to represent sub-grid scale processes may be operating outside the range for which they were designed. On the other hand, statistical downscaling methods use cross-scale relationships that have been derived from observed data, and apply these to climate model data. Statistical downscaling has the advantage of being computationally inexpensive, being able to access finer-scale information than dynamical downscaling, and being applicable to parameters that cannot be directly obtained from global or regional climate model (RCM) outputs (IPCC, 2007). The dynamical downscaling methods have been widely used in China (Gao et al., 2002, 2003, 2006). The current study will focus on the research and application of statistical downscaling methods in China.

In general, any successful statistical downscaling method should satisfy three conditions: (1) the links between predictands and predictors are strong; (2) the predictor variables are well simulated by the GCMs; and (3) the relationship between predictands and predictors does not change with time, and it remains valid in a changed future climate (Fan et al., 2005). According to the second condition, the GCM's ability to represent the large-scale structures associated with the predictors has a relevant role to play in the statistical downscaling process. Zhou and Yu (2006) examined the variations of surface air temperature over China and the globe in the 20th century using 19 coupled climate models driven by historical natural and anthropogenic forcings. They found that most models perform well in simulating both the global and the Northern Hemispheric mean temperature evolution in the 20th century, but few models can produce the summertime cooling over the middle part of eastern China.

Fan et al. (2007) firstly adopted the multiple linear regressions (MLRs) of predictor principle components (PCs) in the statistical downscaling research, but they haven't evaluted the skill of the statistical downscaling method in simulating the warming trend of temperature in China. Fan (2009) found that MLRs of PCs in the statistical downscaling procedure can produce weaker warming in summer than in winter, with similar annual cycles to the observation but different from the HadCM3 output. Besides, the response of the downscaled annual mean (particularly summer) temperature to an enhanced greenhouse effect was weaker than that directly from the HadCM3.

This study will analyze in detail the long-term trend of temperature in January and July 1961-2006 by using statistical downscaling and try to find out the reasons behind. Huth (2002) demonstrated that large-scale free atmospheric temperature and related variables are more informative predictors of local daily mean surface temperature than large-scale circulation fields, and the best results are achieved if one temperature field together with one circulation field are used as predictors. Fan (2006) and Fan et al. (2007) also reported that the best predictor sets are 850 -hPa temperature T850 and H850+T850 ("H" denotes geopotential height). Especially, compared to the former (T850), the latter (H850+T850) shows more physical meanings for the January and July temperature prediction in northern China.

The aims of this study are 1) to check the skill of the statistical downscaling method in simulating the long-term trend of temperature by using T850 and H850+T850 as predictors, respectively; 2) to address the question whether the statistical downscaling can reproduce the observed surface temperature even if the related GCM has a low skill in producing the long-term trend of upper-air temperature; and 3) to compare the differences between the projected EOF analysis and the common EOF analysis (Benestad, 2001, 2002a, b) in projecting the long-term trend of temperature. The data used are introduced in Section 2. Section 3 de- 
scribes the methods employed. Section 4 shows the results, followed by Section 5 that discusses and summarizes the study.

\section{Data}

The predictand fields are the monthly mean surface air temperature at 48 stations in northern China from 1961 to 2006 (Fig. 1). The data were obtained from the China Meteorological Data Sharing Service System. The dataset was processed with quality control and the statistic values were examined with time and internal consistency checking. Some error data were corrected (see the website http://data.cma.gov.cn for details). Only those station records that were complete for the whole time period were used for the analysis. T850 and H850+T850 are employed as large-scale predictor variables in January and July. The large-scale climatic predictors used in this study are derived from the NCEP/NCAR reanalysis data with a resolution of $2.5^{\circ}$ in latitude and longitude for January and July from 1961 to 2006. The GCM data are from HadCM3 under the 20C3M and SRES A1B scenarios for the two sub-intervals 1961-1999 and 2050-2099 and ECHAM5 under the same scenarios for the two sub-intervals 1961-2000 and 2051-2100. The data are available at the IPCC web site (http://ipcc-ddc.cru.uea.ac.uk). Both predictors and predictands are normalized using their respective 1961-1990 mean and standard deviation in all further analyses.

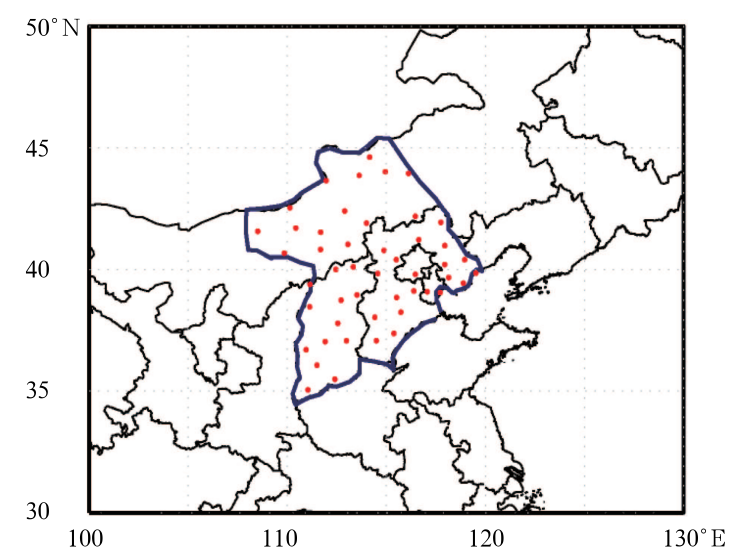

Fig. 1. Research area and locations of the 48 observation stations (dots).

\section{Methodology and initial results}

\subsection{Statistical downscaling models}

MLRs of PCs are used to link the monthly mean temperature to the large-scale climatic predictors based on their historical observations. Before using MLRs, EOF analysis is performed as the first step. For the combinations of two predictors, such as H850+T850, EOF analysis with the two fields combined spatially is used (Bretherton et al., 1992).

The downscaling utilizes a stepwise screening procedure that aims to minimize the Akaike information criterion to obtain the skillful PCs (Wilks, 1995). The different choices of the number of PCs of each predictor variable have been made when they are put into the stepwise regression equation to obtain the optimum linear regression models for each station in January and July.

The downscaling method is also evaluated within the cross-validation framework, where one-year data are excluded from the predictand dataset, and the regression model is built with the remaining 45-yr preditand dataset, and then one prediction is made for the excluded year. This is repeated for all years to yield 46-yr predictions for validation. The resulting 46-yr predictions are then verified against the corresponding 46-yr observations.

In this study, the difference between the mean values of the second and the first half part of the temperature time series is defined as a long-term trend of temperature. Huth $(2002,2004)$ argued that there is a paradox in the downscaling, as the models are calibrated with short-term variations and used to make predictions for long-term changes. He argued that the two series may have a very high correlation but different long-term behaviors such as trend (Benestad et al., 2008). Thus, besides the explained variance, the area-averaged absolute errors of the long-term trend of temperature between the estimates from the statistical models and the observations are also used as a skill score to validate the performance of the statistical models.

The explained variances of cross-validation results using T850 and H850+T850 as predictors under different PCs as inputs to the statistical models are shown 
in Table 1. It is seen that the performance of $\mathrm{H} 850+$ T850 becomes a bit better than that of T850 with the number of PCs increasing (Note that the larger the variance, the better the performance). The areaaveraged absolute errors in the long-term temperature trend between cross-validation results and the observation as given in Table 2 also show the same result (Note that here the smaller the error, the better the performance).

According to values of the area-averaged absolute errors of the long-term temperature trend, the optimal statistical models are selected for each station and for January and July. Tables 3 and 4 show that using the first three PCs as inputs to statistical downscaling models could get the smallest absolute errors between the trends of downscaled temperature and the observation in July for both HadCM3 and ECHAM5. In general, the absolute errors increase as the number of PCs go larger (except for HadCM3 using H850+T850 in January), so the present temperature change is estimated by using the first three PCs as inputs to the statistical models and the results are shown in Fig. 2. We can see that HadCM3 and ECHAM5 could not well simulate the warming trend in northern China in January (Fig. 2). Comparison of the results in Table 3 and 4 (or the curves in Figs. 2a and 2b) reveals that statistical downscaling using the first three PCs of ECHAM 5 T850 and H850+T850 as predictors could better estimate the warming trend as the downscaling has correctly modelled the trend of the PCs associated

Table 1. Explained variances (\%) of cross-validation results to the observation using T850 or H850+T850 as predictors under different numbers of PCs

\begin{tabular}{lcccccc}
\hline \multirow{2}{*}{ Month } & \multirow{2}{*}{ Predictor } & \multicolumn{5}{c}{ Number of PCs } \\
\cline { 3 - 7 } & & 3 & 5 & 10 & 15 & 20 \\
\hline \multirow{2}{*}{ January } & T850 & 50.2 & 51.6 & 59.5 & 68.9 & 70.5 \\
\multirow{2}{*}{ July } & H850+T850 & 48.1 & 51.1 & 63.4 & 65.4 & 70.9 \\
& T850 & 42.4 & 55.0 & 64.2 & 67.3 & 70.3 \\
& H850+T850 & 43.9 & 53.3 & 65.9 & 69.2 & 71.8 \\
\hline
\end{tabular}

Table 2. The area-averaged absolute errors $\left({ }^{\circ} \mathrm{C}\right)$ in the long-term temperature trend between the cross-validation results and the observation using T850 or H850+ T850 as predictors under different numbers of PCs

\begin{tabular}{lcccccc}
\hline \multirow{2}{*}{ Month } & \multirow{2}{*}{ Predictor } & \multicolumn{5}{c}{ Number of PCs } \\
\cline { 3 - 7 } & & 3 & 5 & 10 & 15 & 20 \\
\hline \multirow{2}{*}{ January } & T850 & 0.467 & 0.489 & 0.360 & 0.169 & 0.134 \\
\multirow{2}{*}{ July } & H850+T850 & 0.423 & 0.457 & 0.249 & 0.156 & 0.132 \\
& T850 & 0.512 & 0.345 & 0.262 & 0.266 & 0.234 \\
& H850+T850 & 0.379 & 0.306 & 0.213 & 0.201 & 0.198 \\
\hline
\end{tabular}
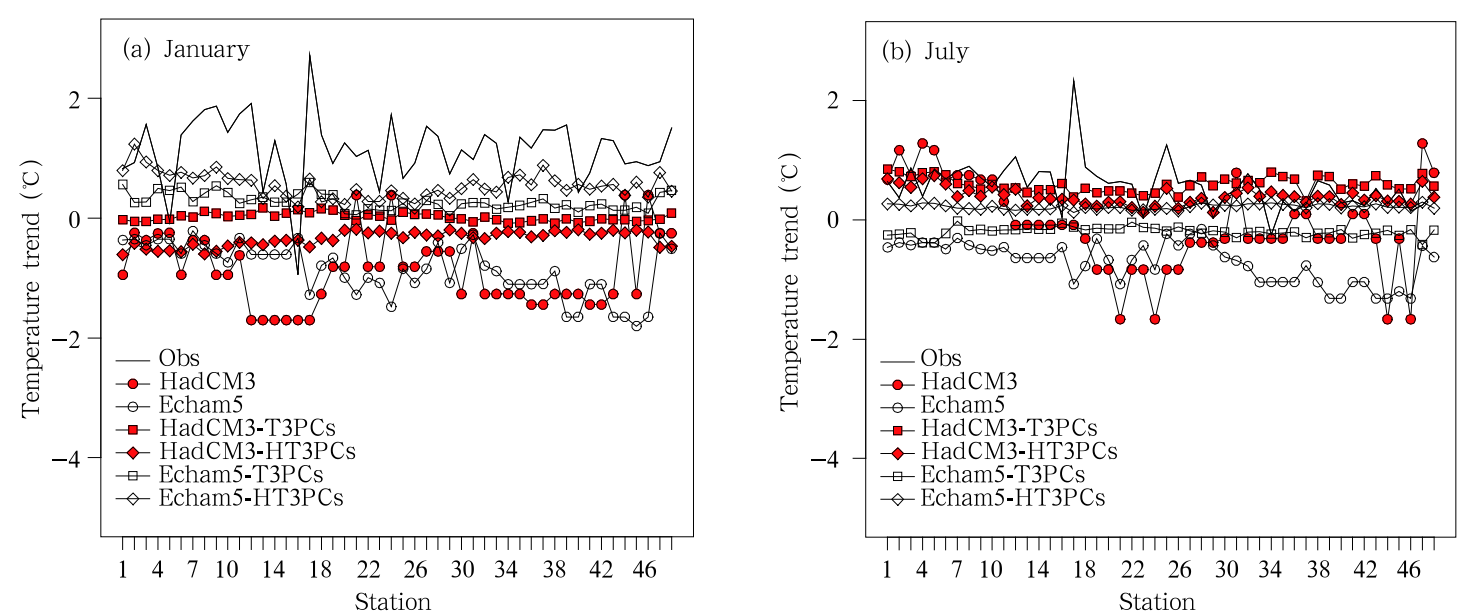

Fig. 2. The present long-term trend of temperature. "T3PCs" denotes the model using the first three PCs of T850 as predictors; "HT3PCs" denotes the model using the first three PCs of H850+T850 as predictors. (a) January and (b) July. 
Table 3. The area-averaged absolute errors $\left({ }^{\circ} \mathrm{C}\right)$ between the statistically downscaled long-term trend of temperature derived from HadCM3 and the observed ones using different numbers of PCs in the statistical models

\begin{tabular}{|c|c|c|c|c|c|c|}
\hline \multirow{2}{*}{ Month } & \multirow{2}{*}{ Predictor } & \multicolumn{5}{|c|}{ Number of PCs } \\
\hline & & 3 & 5 & 10 & 15 & 20 \\
\hline \multirow{2}{*}{ January } & $\mathrm{T} 850$ & 1.15 & 1.14 & 1.36 & 1.34 & 1.29 \\
\hline & $\mathrm{H} 850+\mathrm{T} 850$ & 1.49 & 1.48 & 1.39 & 1.40 & 1.29 \\
\hline \multirow{2}{*}{ July } & T850 & 0.31 & 0.43 & 0.44 & 0.49 & 0.56 \\
\hline & $\mathrm{H} 850+\mathrm{T} 850$ & 0.30 & 0.36 & 0.34 & 0.35 & 0.40 \\
\hline
\end{tabular}

Table 4. As in Table 3, but for ECHAM5

\begin{tabular}{lcccccc}
\hline \multirow{2}{*}{ Month } & \multirow{2}{*}{ Predictor } & \multicolumn{5}{c}{ Number of PCs } \\
\cline { 3 - 7 } & & 3 & 5 & 10 & 15 & 20 \\
\hline \multirow{2}{*}{ January } & T850 & 0.93 & 0.99 & 1.31 & 1.57 & 1.64 \\
\multirow{3}{*}{ July } & H850+T850 & 0.69 & 0.70 & 1.15 & 1.20 & 1.32 \\
& T850 & 0.76 & 0.99 & 0.94 & 1.02 & 0.90 \\
& H850+T850 & 0.40 & 0.38 & 0.42 & 0.47 & 0.47 \\
\hline
\end{tabular}

with the regional warming, while HadCM3 has generated a warming trend with larger biases due to incorrectly modelled trend of the first three PCs. In January, the results of $\mathrm{H} 850+\mathrm{T} 850$ are better than those of T850 for ECHAM5. In July, the original HadCM3 and ECHAM5 temperature outputs contain no positive temperature anomalies while statistical downscaling models using HadCM3 variables as predictors have largely improved the model skills in predicting temperature changes in July and using H850+T850 as predictors produces better results.

\subsection{Statistical downscaling implementation}

The optimal statistical downscaling models in January and July were applied to the HadCM3 and ECHAM5 IPCC 20C3M and A1B scenarios. Before this, the two GCMs' output data were linearly regridded to the $2.5^{\circ} \times 2.5^{\circ}$ latitude-longitude grid. The data to be used as predictors were standardized by the mean and standard deviation with respect to the 1961-1990 GCM outputs. This procedure ensured that the values downscaled from the GCM runs were free of the GCM model bias.

Then, in order to ensure a good correspondence between the GCM simulated EOFs and the observed ones, two methods were used. One was that GCM predictor anomalies were projected onto the observed EOFs by using the projected EOF analysis; the other was to find the common EOF of the observation and the GCM output by carrying out a common EOF analysis. These two methods were performed using the tools in the $\mathrm{R}$ package developed by the authors themselves. The GCM projected PCs and the GCM PCs obtained by the common EOF analysis were incorporated into the downscaling models that had been developed on the basis of the observations. The statistical downscaling models' outputs were first inflated by the inverse of their standard deviations derived from 1961 to 1990 , analogical to the observations, in order not to lose variance, and then were destandardized by the observed mean and standard deviation. This approach enabled the downscaling output to be adjusted to the observed mean and standard deviation (Winkler et al., 1997) and resulted in larger fractions of temperature variance explained by the downscaling model (Huth, 2004).

\section{Results}

\subsection{Comparison of $\mathbf{T 8 5 0}$}

The skill of the statistical downscaling models depends strongly on the ability of GCMs in simulating the large-scale predictor variable. It is thus necessary to evaluate the GCMs' performance in producing the long-term trend of T850. It can be seen from the T850 changes in the NCEP/NCAR reanalysis in Figs. 3a,b that regional warming occurs all over the predictor domain in January (Fig. 3a), and only a strong warm- 
ing center appears within $35^{\circ}-40^{\circ} \mathrm{N}, 110^{\circ}-125^{\circ} \mathrm{E}$ while cooling dwells in the northwest part of the domain in July (Fig. 3b). Both HadCM3 and ECHAM5 failed to simulate the January (Figs. 3c, e) and July (Figs. 3d, f) T850 changes. Compared with the reanalysis, these two models have produced a cooling trend in
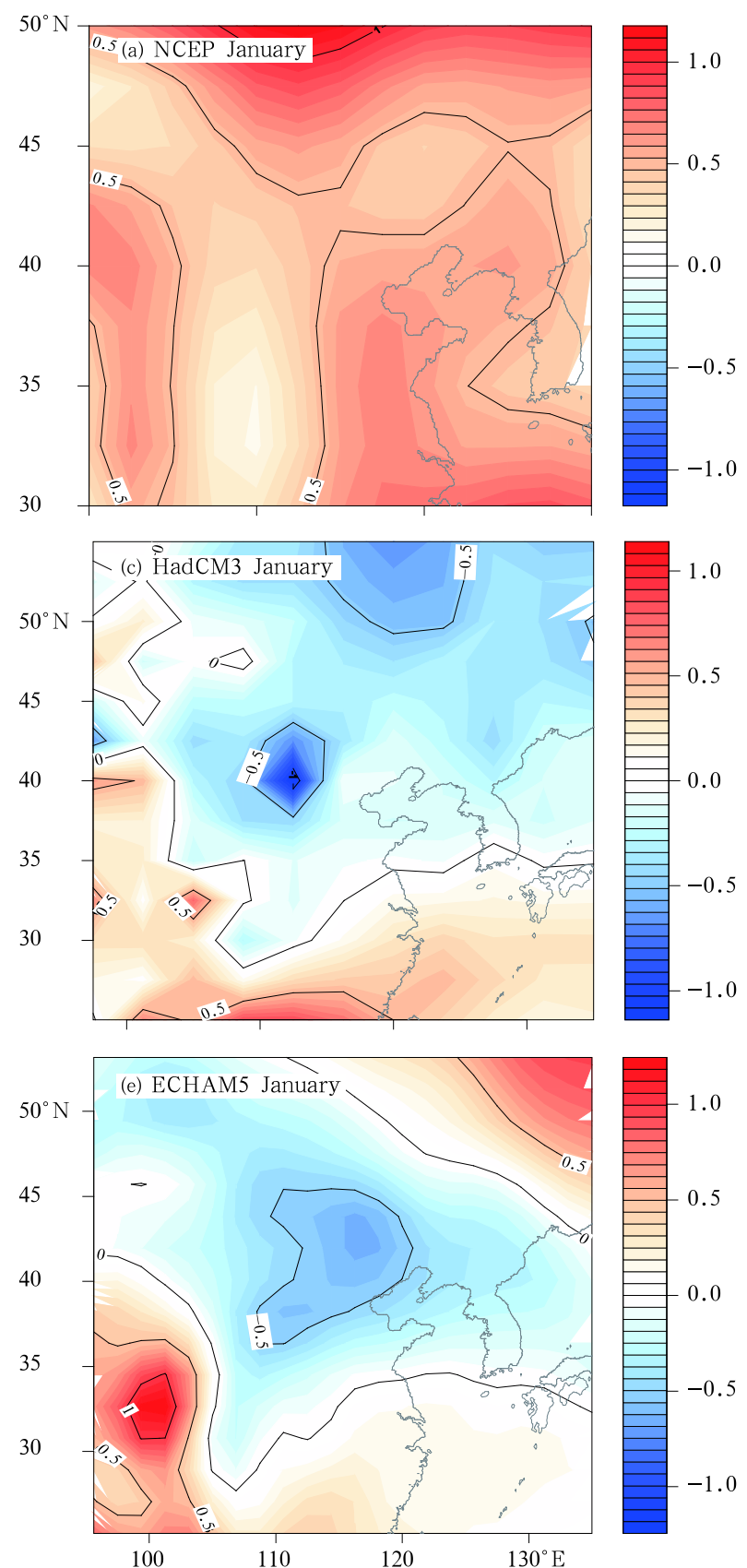

most parts of the domain in January (Figs. 3c, e) and a totally different pattern of the temperature change in July (Figs. 3d, f).

Figure 4 shows that in January, the H850+T850 field exhibits similar features to the first EOF patterns of T850, and is correlated with changes of geopotential
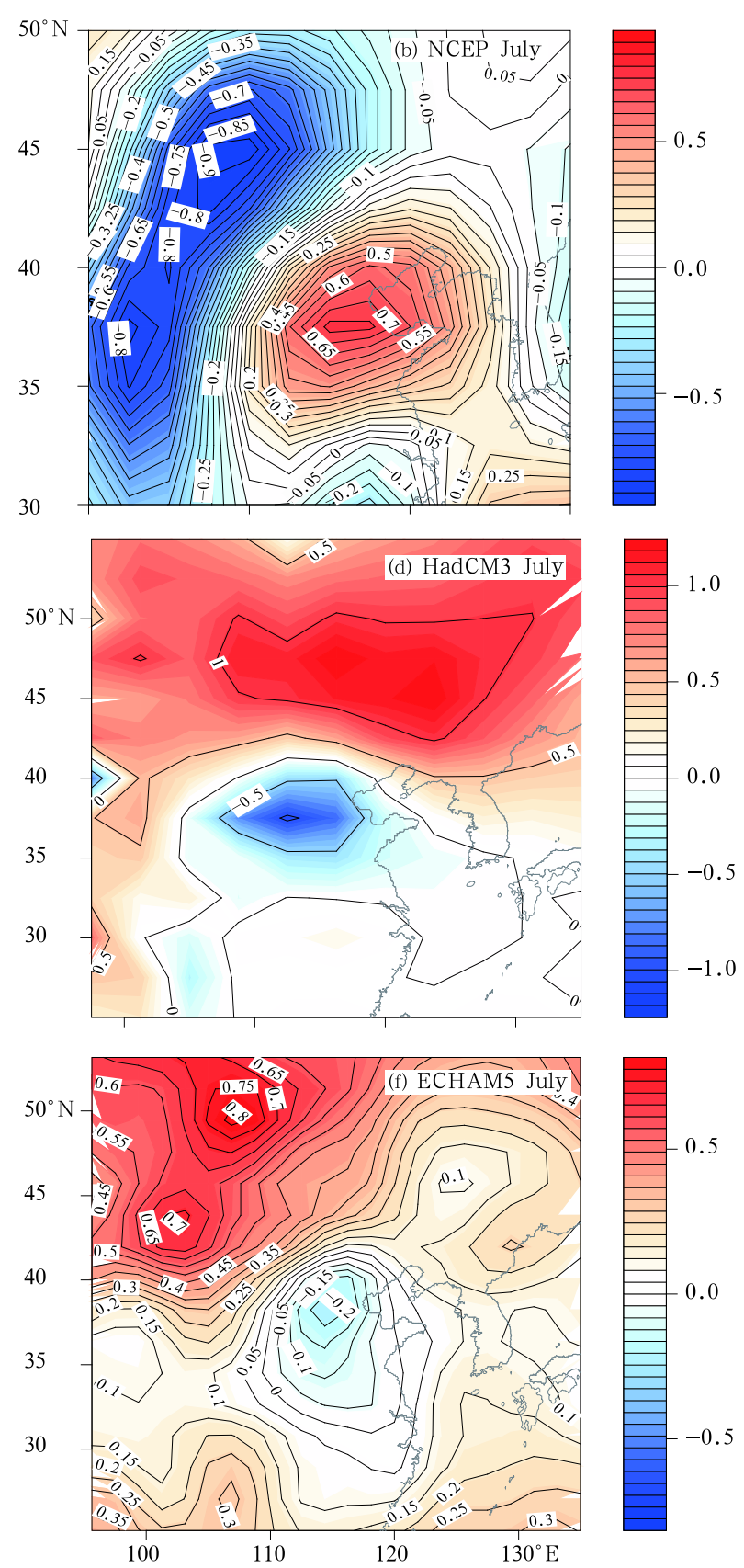

Fig. 3. Comparison of T850 trend $\left({ }^{\circ} \mathrm{C}\right.$ ) derived from NCEP (upper panels; 1981-2000 minus 1961-1980), HadCM3 (middle panels; 1980-1999 minus 1960-1979), and ECHAM5 (lower panels; 1981-2000 minus 1961-1980). Left panels are for January and right panels for July. 

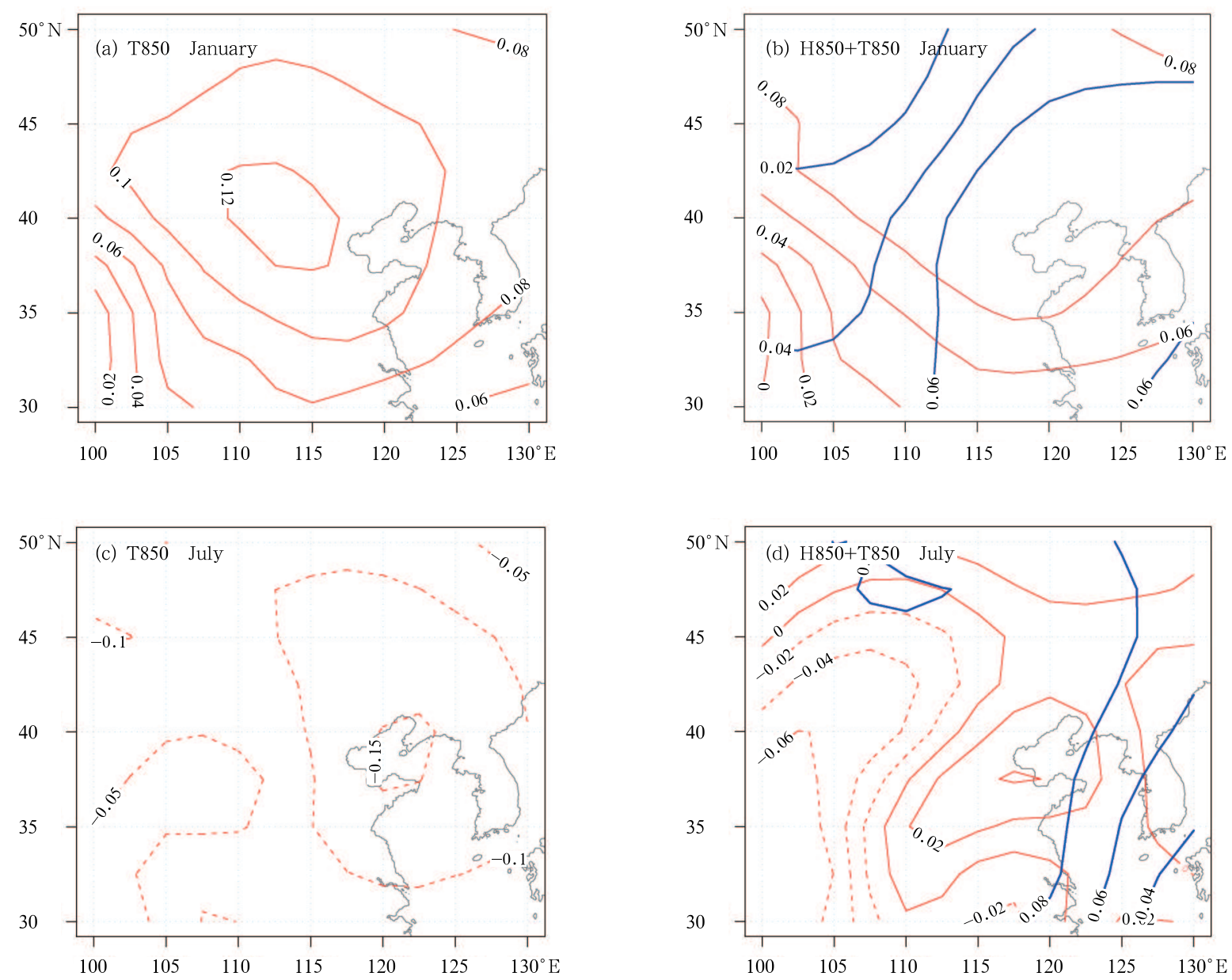

Fig. 4. The first EOF patterns of T850 (left panels) and H850+T850 (right panels) of NCEP reanalysis in January (a, b) and July (c, d). The red lines are for T850 and the blue lines for H850.

height at $850 \mathrm{hPa}$. The EOFs of the combined field of H850 and T850 could well explain the related physical processes. For example, the first EOF pattern represents a regional warming (Fig. 4a) that resembles the positive T850 anomaly in the NCEP reanalysis (Fig. 3a) and accompanies the regional positive change of H850 (Fig. 4b). The first PCs of T850 and H850+T850 in January are compared in Fig. 5a. It is found that they are well consistent with each other. Thus, it is inferred that the first EOF pattern with regional warming accompanied by regional increase of H850 can largely determine the long-term trend of T850. The second EOF pattern shows a north-south variation of temperature associated with the increase of H850. The third EOF represents east-west changes of temperature associated with the east-west changes of H850 (figure omitted).

In July, the regional warming appears in the second EOF pattern of H850+T850 (figure omitted). The PCs associated with the regional warming have also been compared between T850 and H850+T850 (Fig. $5 \mathrm{~b})$ and they are well consistent with each other, similar to the situation in January. The regional warming also accompanies a regional increase in H850. However, it should also be noted from Fig. 4d that the first EOF pattern of H850+T850 is similar to the trend of T850 shown in Fig. 3b. One important finding is that the pattern of long-term trend of T850 in July is observed in the first EOF pattern of H850+T850, not in the first EOF pattern of T850. 

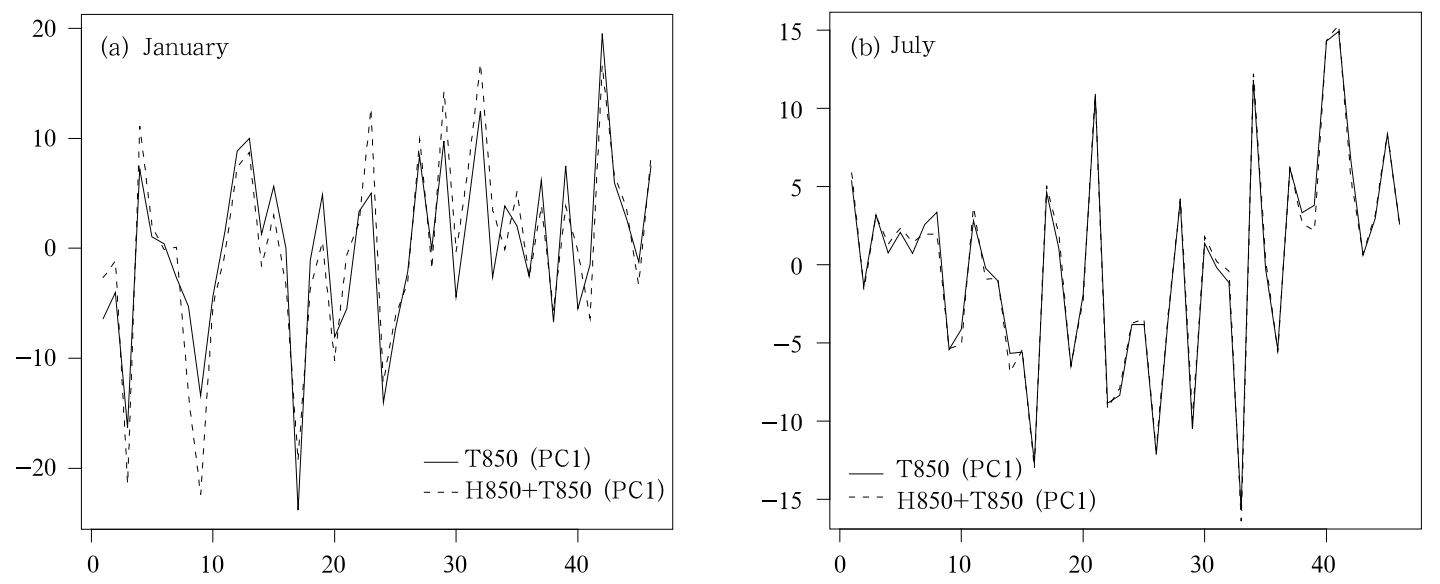

Fig. 5. Comparison of the PC corresponding to the regional warming between H850+T850 and T850 in (a) January and (b) July.
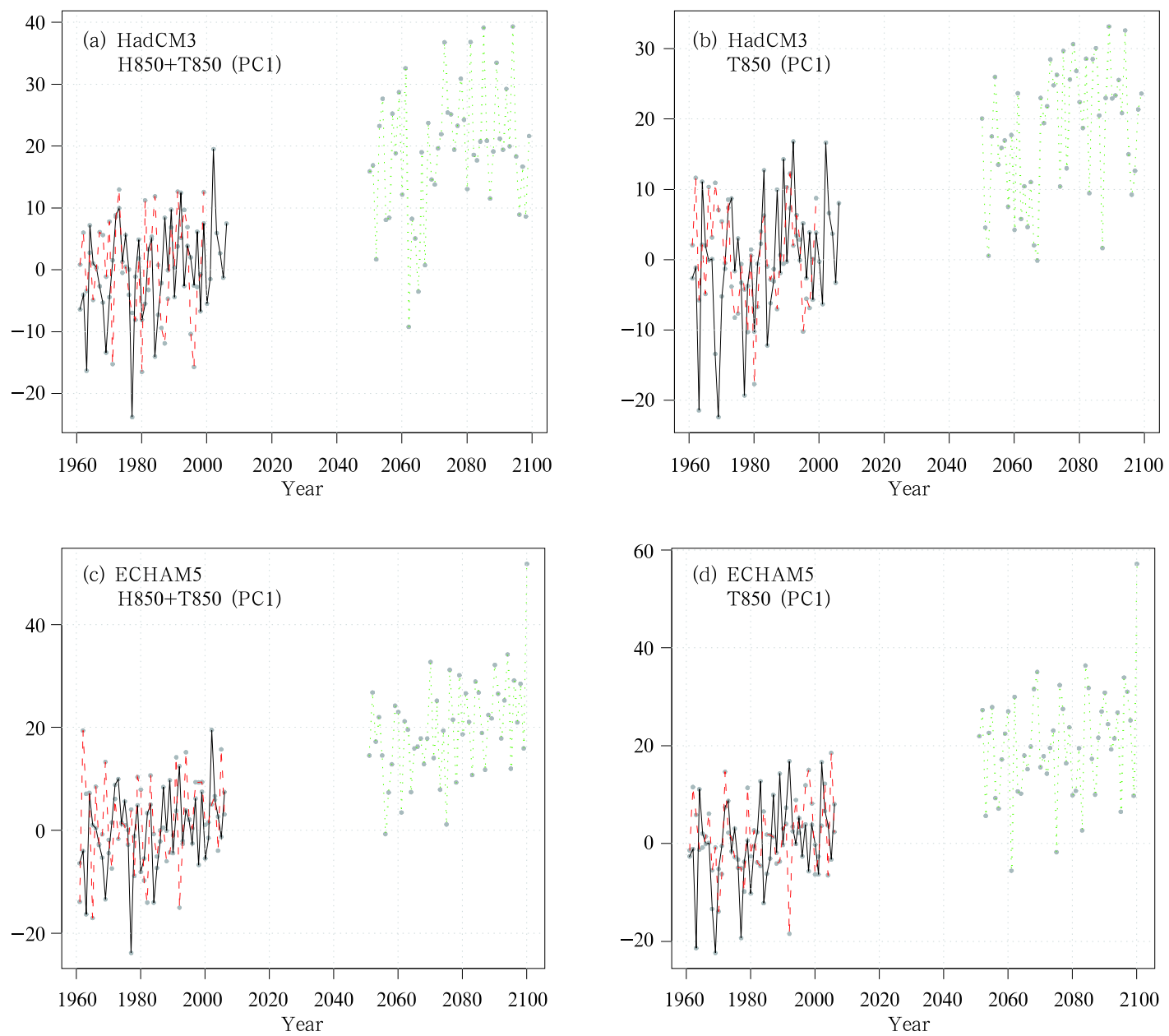

Fig. 6. The first PCs of H850+T850 (left panels) and T850 (right panels) from HadCM3 (upper panels) and ECHAM5 (lower panels) projected onto NCEP in January. The black solid lines are for NCEP, the dashed lines for the two GCMs, the red lines for the present climate, and the green lines for future climate. 

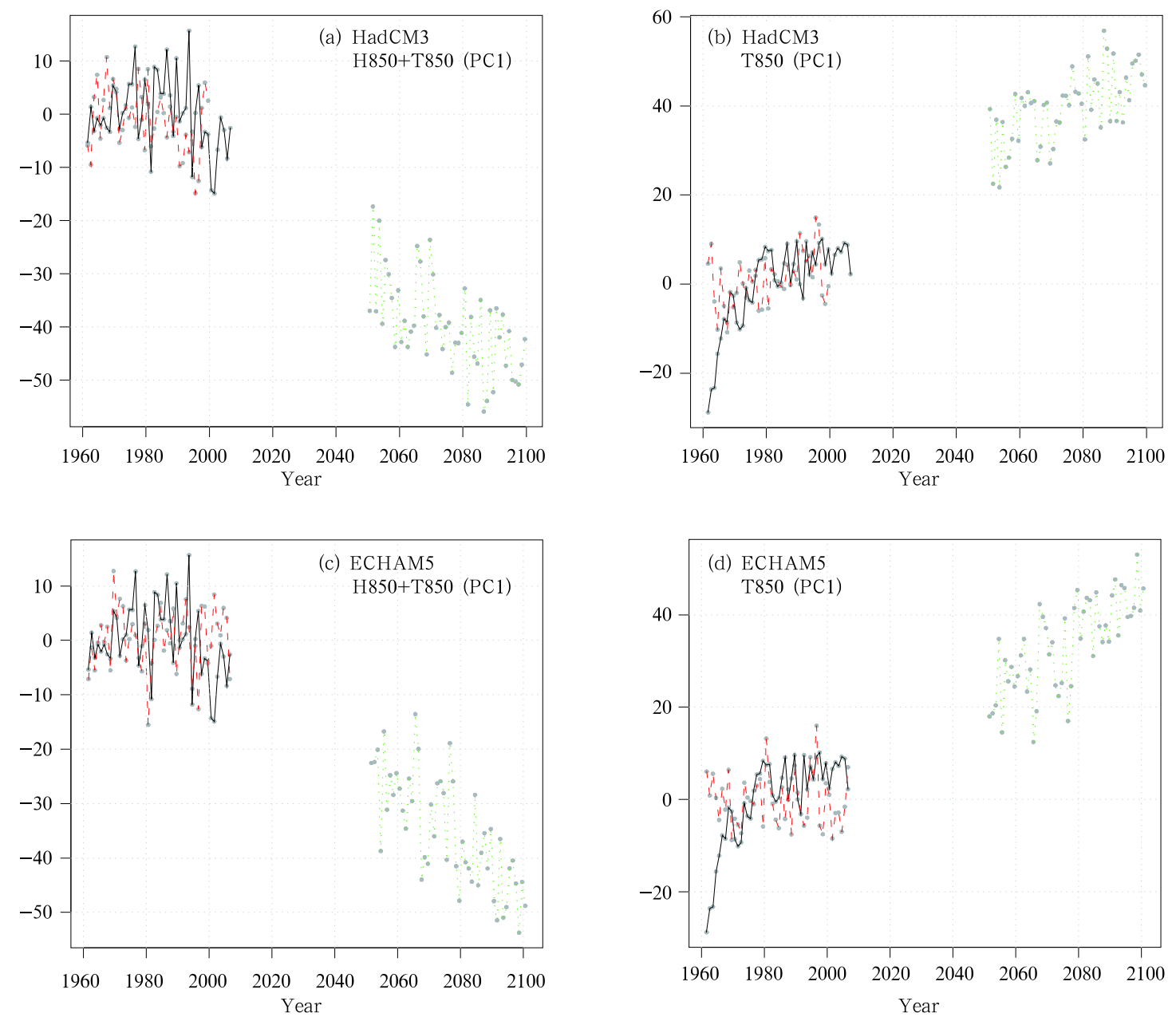

Fig. 7. As in Fig. 6, but for July.

\subsection{Present trend of temperature from the statistical downscaling}

The two predictor sets of T850 and H850+T850 derived from both HadCM3 and ECHAM5 have been projected onto the observed EOF patterns of T850 and H850+T850 using the projected EOF analysis. For H850+T850, H850 and T850 from each GCM should be combined spatially before being projected. The first PCs from HadCM3 and ECHAM5 are shown in Figs. 6 and 7. In the present climate, HadCM3 cannot simulate a warming trend in the first PCs of T850 and H850+T850 in January, while ECHAM5 can produce a more consistent trend. In July, HadCM3 gives an increasing trend in the first PC of T850 (Fig. 7b) and the second PC of H850+T850 (not shown), and also gives a more consistent increase in the first PC of
H850+T850 (Fig. 7a), whereas the first two PCs from ECHAM5 could not simulate the temperature trend.

\subsection{Future trend of temperature}

We now compare the trends of PCs associated with EOF patterns of the regional warming in Fig. 8. In January, no matter which EOF analysis (the projected or the common one) is used, PCs have a similar warming trend. For the projected EOF analysis, the PC of T850 has a little stronger warming trend than that of H850+T850. In July, the PC of the projected T850 has a similar trend to those of H850+T850 and T850 derived from the common EOF analysis. However, it is noted that the $\mathrm{PC}$ of projected H850+T850 associated with the regional warming has a much weaker warming trend than the others. 

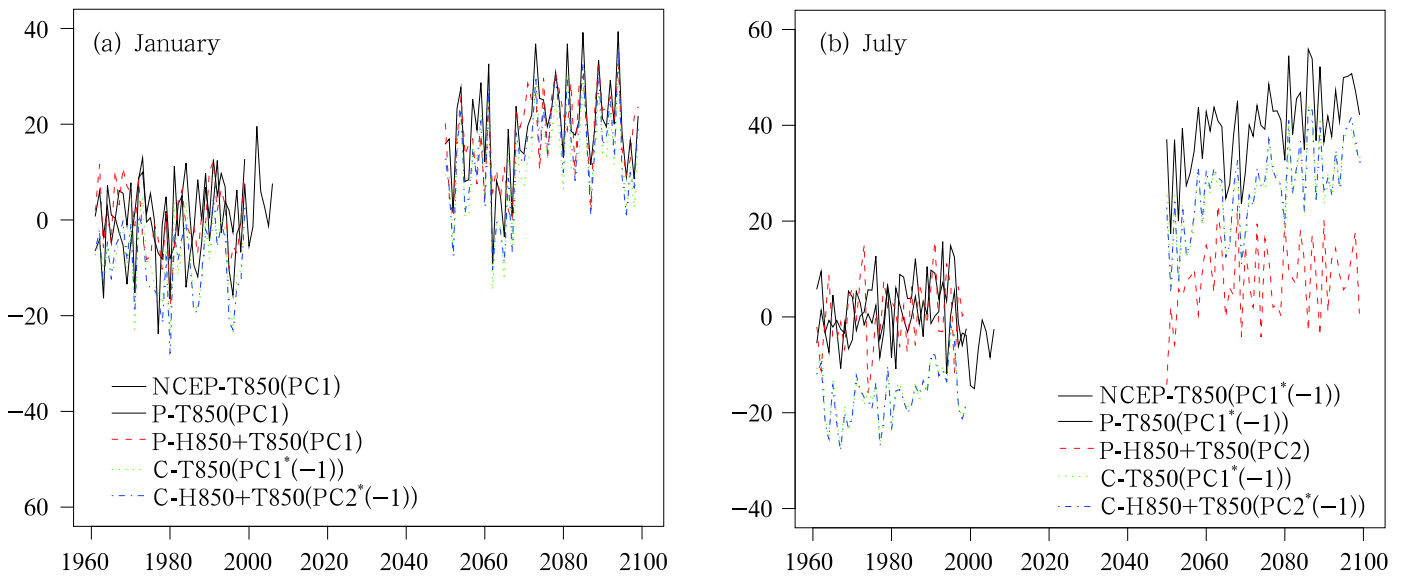

Fig. 8. Comparison of PCs associated with the regional warming pattern from HadCM3. "P" denotes PC using the projected EOF method; "C" denotes PC using the common EOF method. (a) January and (b) July.
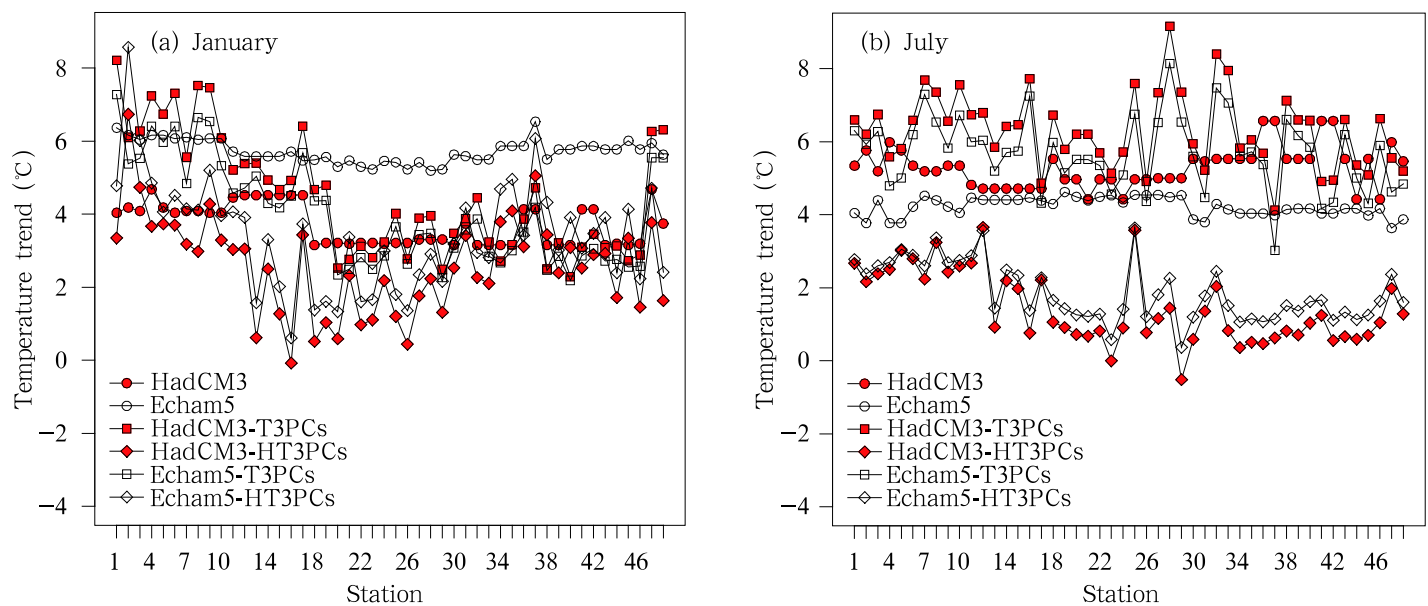

Fig. 9. Future long-term temperature trend estimated by the projected EOF method. The difference of the mean temperature between 1961-1990 and 2070-2099 for HadCM3 and 2071-2100 for ECHAM5 is shown. "T3PCs" denotes the method using the first three PCs of T850 as predictors; "HT3PCs" denotes the method using the first three PCs of H850+T850 as predictors. (a) January and (b) July.
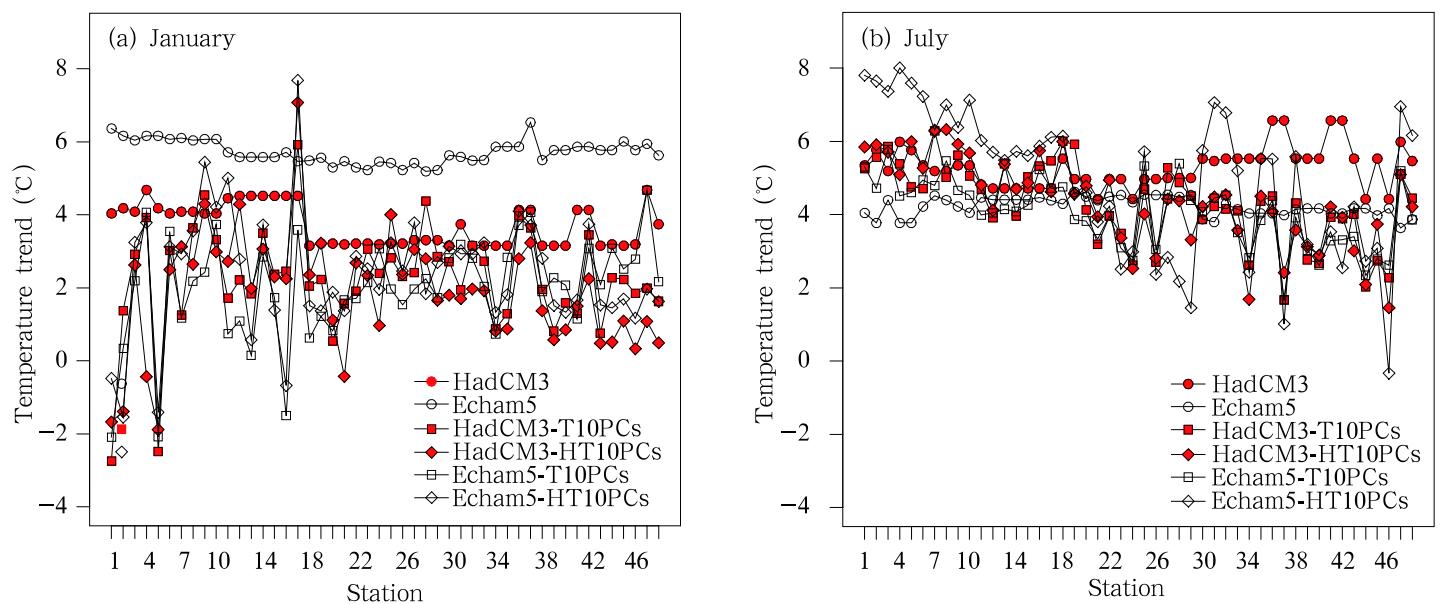

Fig. 10. Future long-term temperature trend estimated by the common EOF method. The difference of the mean temperature between 1961-1990 and 2070-2099 for HadCM3 and 2071-2100 for ECHAM5 is shown. "T10PCs" denotes the method using the first 10 PCs of T850 as predictors; "HT10PCs" denotes the method using the first 10 PCs of H850+T850 as predictors. (a) January and (b) July. 
In January, the statistical downscaling method produces similar trends of temperature with different large-scale GCMs and different predictor choices (Fig. 9). In July, H850+T850 gives a much lower warming trend than T850. This is probably due to a lower warming trend in the PC of the projected H850+T850. Figure 10 shows the future long-term temperature changes estimated by the common EOF technique. Although both HadCM3 and ECHAM5 simulate obviously different long-term trends of temperature, the statistically downscaled results using two GCM variables as predictors can produce similar longterm temperature change scenarios at most stations. It is also noted that the results from the common EOF technique in July have much stronger warming trends than those in January.

\section{Summary}

In this study, the statistical downscaling models using the predictor of H850+T850 rather than T850 gives a better skill for producing the long-term trend of temperature at most of the 48 stations in northern China. This agrees with the results from Fan et al. (2007) and Huth (2002). At the same time, the EOF analysis with the two fields combined spatially may have more physical meanings than that of a single field (Fan et al., 2007). For instance, according to the results of EOF analysis of $\mathrm{H} 850+\mathrm{T} 850$, the regional warming trends were mainly accompanied by increases in geopotential height at $850 \mathrm{hPa}$ in January and July. Moreover, the temperature change at $850 \mathrm{hPa}$ has a weaker signal in the EOF pattern associated with the regional warming in July than in January. Since the EOF analysis of H850+T850 highlights the first EOF pattern, a center of increase in temperature is located within $35^{\circ}-40^{\circ} \mathrm{N}, 110^{\circ}-125^{\circ} \mathrm{E}$, accompanied by an increase in geopotential height at $850 \mathrm{hPa}$, and more importantly, this spatial pattern is similar to the long-term observed temperature change in July in northern China. We may conclude that the temperature change in July is associated with the first combined EOF pattern of H850+T850, not with the EOF pattern of T850. This finding coincides with the results of $\mathrm{Hu}$ et al. (2003) from a comparison between the observed seasonal climate variation and the CMIP2 simulations of 16 models. Their results indicated that the observed long-term variations of winter, spring, and autumn temperatures in China may be associated with increases in greenhouse gas concentrations; however, such a connection is not found for the summer temperature.

In the present study, the combined EOF pattern is likely to be explained by strong and enlarged continental subtropical high, which causes an increase in air temperature through descending motion. Xie et al. (1999) analyzed the probability of occurrences of severe hot weather in northern China during 19501999, and Sun et al. (1999) analyzed the mechanism causing the hot weather in northern China in July using the mecoscale model MM5. They made the same conclusion that hot weather is due to the abnormal strong and enlarged continental subtropical high, which maintains for a long period of time with sub-centers settled over northern China resulting in an increase in air temperature there because of enhanced descending motion.

Although HadCM3 and ECHAM5 have simulated a false trend in long-term temperature change for T850, the statistical downscaling method is able to reproduce the observed long-term temperature change in northern China due to correct simulations of the trend of the first few PC predictors of the GCMs. It is concluded that the results of the statistical downscaling depend incompletely on the direct GCM outputs and can effectively reduce the errors of the GCMs.

By projecting direct GCM outputs onto the observed EOFs of H850+T850, the PC associated with the EOF pattern of the regional warming has a much lower warming rate compared to that only using single T850 as the predictor in July. This indicates that the warming modelled by the two GCMs is never completely attributed to the increase of H850. The statistical downscaling of projected EOF of H850+T850 yields a better agreement with the observed temperature variation and produces a better warming in January than in July. This is completely different from the scenarios based on the direct HadCM3 and ECHAM5 
outputs. The latter suggested more warming in summer than in winter. In this case, the projected EOF analysis generates future long-term temperature trends similar to the historical observations in spite of different GCM outputs. This is also different from the results of Precis (Xu et al., 2006), who showed that temperature in China will become warmer in summer than in winter in the 2080s. This is probably because RCM simulations driven by GCMs will inherit more errors from the GCMs than the statistical downscaling method.

From the temperature scenarios derived by the common EOF analysis, different GCMs could produce similar trends of long-term temperature change. Compared with the results of the projected EOF analysis, the common EOF method produces a warming in July much stronger than in January and are more mutually consistent. It is in good agreement with the direct GCM outputs and RCM simulations, but contrary to the observation. This is likely because the common EOFs derived from both NCEP reanalysis and GCM outputs are mostly determined by GCM predictors rather than those from only the NCEP reanalysis, owing to a much longer time series of GCMs. Compared with the common EOF method, an advantage of the projected EOF method is that it depends more strongly on the historical observations because GCM predictors have been forced to project onto the observed EOFs.

\section{REFERENCES}

Benestad, R. E., 2001: A comparison between two empirical downscaling strategies. Int. J. Climatol., 21, $1645-1668$.

_- 2002a: Empirically downscaled multi-model ensemble temperature and precipitation scenarios for Norway. J. Climate., 15, 3008-3027.

— , 2002b: Empirically downscaled temperature scenarios for northern Europe based on a multi-model ensemble. Climatic. Res., 21(2), 105-125.

—, I. Bauer-hanssen, and D. Chen, 2008: EmpiricalStatistical Downscaling. World Scientific Publishing Company, 300 pp.

Bretherton, C. S., C. Smith, and J. M. Wallace, 1992: An intercomparison of methods for finding coupled patterns in climate data. J. Climate, 5, 541-560.

Fan Lijun, 2006: Statistical downscaling of local and regional climate scenarios over China. Ph. D. dissertation. Institute of Atmospheric Physics, Chinese Academy of Sciences, 32-46.

_ 2009: Statistically downscaled temperature scenarios over China. Atmospheric and Oceanic Science Letters, 2(4), 208-213.

_- Fu Congbin, and Chen Deliang, 2005: Review on creating future climate change scenarios by statistical downscaling techniques. Adv. Earth Sci., 20(3), 320-329.

,$- \ldots$, and — , 2007: Estimation of local temperature change scenarios in North China using the statistical downscaling method. Chinese J. Atmos. Sci., 31(5), 887-897.

$\mathrm{Hu}, \mathrm{Z}$., S. Yang, and R. Wu, 2003: Long-term climate variations in China and global warming signals. Journal of Geophysical Research, 108(D19), 4614, doi: 10.1029/2003JD0033651.

Huth, R., 2002: Statistical downscaling of daily temperature in central Europe. J. Climate, 15, 1731-1742.

—, 2004: Sensitivity of local daily temperature change estimates to the 432 selection of downscaling models and predictors. J. Climate, 17, 640-652.

IPCC, 2007: The IPCC Fourth Assessment Report: Climate Change 2007, 918 pp.

Gao, X. J., Z. C. Zhao, and F. Giorgi, 2002: Changes of extreme events in regional climate simulations over East Asia. Adv. Atmos. Sci., 19, 927-942.

—, Z. C. Zhao, and Y, H. Ding, 2003: Climate change due to greenhouse effects in Northwest China as simulated by a regional climate model. J. Glaciol. Geocryol., 25(2), 165-169.

_ - J. S. Pal, and F. Giorgi, 2006: Projected changes in mean and extreme precipitation over the Mediterranean region from a high resolution double nested RCM simulation. Geophys. Res. Lett., 33, L03706, doi:10.1029/2005GL024954.

Sun, J., H. Chen, S. Zhao, Q. Zeng, Z. Xie, J. Cui, and H. Liu, 1999: A study on the severe hot weather in Beijing and North China. Part II: Simulation and analysis. Climatic and Environmental Research, 4, 334-345.

Wilks, D. S., 1995: Statistical Methods in the Atmospheric Science. Academic Press, 467 pp.

Winkler, J. A., J. P. Palutikof, and J. A. Andresen, 1997: The simulation of daily temperature time series from 
GCM output. Part II: Sensitivity analysis of an empirical transfer function methodology. J. Climate, 10, 2514-2535.

Xie, Z., J. Cui, H. Liu, S. Zhao, J. Sun, H. Chen, and Q. Zeng, 1999: A study on the severe hot weather in Beijing and North China. Part I: Statistics and synoptic case study. Climatic and Environmental Research, 4, 323-333.
Xu, Y., Y. Zhang, E. Lin, W. Lin, W. Dong, J. Richard, H. David, and W. Simon, 2006: Analyses on the climate change responses over China under SRES B2 scenario using PRECIS. Chinese Science Bulletin, 51, 2260-2267.

Zhou, T., and R. Yu, 2006: Twentieth-century surface air temperature over China and the Globe simuated by coupled climate models. J. Climate, 19, 5843-5858. 